\title{
À la recherche de nouvelles catégories pour l'analyse du discours - quand la vulgarisation scientifique passe par les blogs
}

\author{
Sandrine Reboul-Touré ${ }^{1}$ \\ EA 7345 Clesthia, Langage, systèmes, discours, Maison de la Recherche, 4 rue des Irlandais, Sorbonne nouvelle, Paris, \\ France
}

\begin{abstract}
Résumé. La vulgarisation scientifique depuis les Entretiens sur la pluralité des mondes de Fontenelle s'est glissée dans différents genres, entretien, poésie, roman, bande-dessinée, article de revues, article de journal pour l'écrit mais aussi via des médias comme la télévision ou la radio. Le support internet a relancé la circulation des discours de vulgarisation scientifique, notamment au travers des sites ouverts par les revues papier comme La Recherche ou Science \& vie mais aussi de sites spécifiquement conçus pour le support internet comme Futura Sciences, en France. Avec internet se développe une "communication numérique écrite », avec notamment les blogs qui offrent des facilités de publication et d'interaction. Certains internautes se sont emparés des blogs pour vulgariser la science. Face à ces nouvelles formes discursives, nous nous interrogeons sur l'extension du concept de genre. Par ailleurs, les blogs se présentent comme des discours hybrides et dans le cadre de l'analyse du discours, nous cherchons à revisiter certaines catégories d'analyse voire à prospecter vers de nouvelles catégories.
\end{abstract}

\begin{abstract}
In Search of New Categories for Discourse Analysis - Popular Science through Blogs. Popular science since Fontenelle's Entretiens sur la pluralité des mondes uses different genres: interviews, poetry, novels, comic strips, magazine articles, newspaper articles, but also via media such as television and radio. The Internet has revived the circulation of popular science discourse, particularly through sites opened by journals such as La Recherche or Science \& Vie, but also through sites specifically designed for the Internet such as Futura Sciences, in France. Thanks to the Internet, a "written digital communication" is developing, with blogs that offering easy publication and interaction. Some Internet users have taken up blogs to popularise science. In front of these new discursive forms, questions are being asked about the extension of the concept of 'genre'. Moreover, blogs are presented as hybrid discourses and in the framework of discourse analysis, we revisit some categories of analysis or prospect towards new categories.
\end{abstract}

\section{Introduction}

L'analyse des discours de transmission des connaissances constitue un « terrain discursif » exploré depuis de nombreuses années, notamment au sein du groupe de recherche du CEDISCOR $^{1}$ de la Sorbonne nouvelle. Deux orientations ont été distinguées pour les discours de transmission des connaissances, d'une part, la transmission des connaissances institutionnelles, dans un cadre formel, notamment celui de l'Ecole, des petites sections à l'université. On est alors du côté de l'enseignement qui doit être noté par des évaluations avec l'observation de résultats effectifs de l'apprentissage. D'autre part, une transmission des connaissances non-formelle qui passe par des médias qui étaient au départ peu académiques, la télévision, la radio, la presse spécialisée mais aussi la presse d'actualité et aujourd'hui, internet. Ici, les connaissances sont proposées, souvent via des vulgarisateurs, et le public choisi d'acquérir ce qu'il souhaite sans résultat imposé. On trouve donc au moins deux voies, celle du faire savoir et celle du faire connaître.

Nous nous intéressons ici à la transmission de la science dans des lieux non-formels. Ce processus donne naissance à des discours qu'on peut faire entrer dans le champ de la vulgarisation scientifique et la vulgarisation scientifique peut être considérée comme un type de discours. En effet, on a identifié des propriétés linguistiques caractéristiques de ces discours : ils sont porteurs d'hétérogénéités énonciatives (Authiez-Revuz 1984) car ils font entendre au moins deux discours, les discours sources (ceux des spécialistes) et les discours seconds (ceux qui s'adressent à un public moins averti). Cette hétérogénéité est saillante via de nombreuses marques formelles, d'une part, celles des discours rapportés et on identifie de nombreuses voix (chercheurs, vulgarisateurs, lecteurs/auditeurs) - du moins au début de la production des textes de vulgarisation scientifique. D'autre part, des glissements discursifs (des « entailles » (Mongi et alii éds 2014) sont caractéristiques de la reformulation, notamment autour des mots spécialisés. Ce concept a été proposé par Peytard qui considère la reformulation comme une des voies de l'altération, de «l'autrement dit/l'autrement fait 》 (Peytard 1993). On peut alors revisiter la reformulation en parlant plutôt d'une catégorie descriptive pour l'analyse du discours (Moirand

\footnotetext{
${ }^{1}$ sandrine.reboul-toure@sorbonne-nouvelle.fr
} 
2012, Reboul-Touré 2014) : « la reformulation fait converger une analyse lexicale associée à la dynamique d'une analyse discursive : c'est autour des mots spécialisés de la science que se mettent en place des réécritures, des équivalences sémantiques, des désignations coréférentielles avec une large exploration des relations sémantiques (hypo/hyperonymie, quasi-synonymie, métaphore...) pour mieux appréhender le sens » (Reboul-Touré, à paraître). L'hétérogénéité peut aussi se combiner avec la reformulation et constituent des caractéristiques discursives internes.

Considérer la vulgarisation scientifique comme un type de discours (Adam 2001) permet peut-être d'identifier différents genres comme la nouvelle, l'entretien, la poésie, la bande dessinée, formes textuelles dans lesquelles peut se glisser la transmission de connaissances ; à moins que ces « coquilles textuelles » porteuses d'éléments issus de la science soient plutôt à envisager comme "scénographie » (Maingueneau 1998). On voit que le concept de genre demande à être retravaillé notamment face à des discours hybrides, par exemple, lorsqu'on cherche à diffuser la science au travers de poèmes ou bien de bandes dessinées. Ce questionnement apparaît aussi lorsqu'on se propose d'analyser des objets discursifs qui n'existaient pas auparavant, comme ces nouveaux genres (peut-on parler de genre ?) qui émergent sur le support de l'internet, notamment, les blogs. Lorsqu'on analyse les discours issus d'internet, on est amené à forger de nouvelles catégories pour l'analyse.

J'ai comparé d'une part des blogs de vulgarisation scientifique en France depuis 2013 (Reboul-Touré 2015) et au Brésil


des sciences en France et Agence Science-Presse 4 pour les « blogues » au Québec - afin de mettre en relation des aires culturelles différentes bien que partageant une langue commune (voir les adresses des portails rassemblant différents blogs dans « Corpus »). Cette transmission s'inscrit dans des choix politiques puisque développer la culture scientifique auprès du grand public participe à l'émancipation des citoyens. En effet, les intervenants, dans la chaîne de la transmission de la science, se diversifient et on trouve de nouvelles figures comparativement au " $3{ }^{\mathrm{e}}$ homme » qui était le vulgarisateur, dans les années 1980. De nouvelles questions surgissent alors pour l'analyse du discours. Peut-on parler de genres pour les blogs de science? Qu'en est-il des nouvelles figures comme celles de l'amateur ? Est-ce que la notion de « sphère d'activité langagière » est plus adéquate que celle de «communauté discursive » pour décrire ces nouvelles configurations discursives?

\section{Le blog de vulgarisation scientifique : stabilisation d'un genre?}

« Site internet animé par un individu ou une communauté qui s'exprime régulièrement dans un journal, des billets », telle est la définition proposée par le Petit Robert 2020. Cet anglicisme, de web et log (carnet de bord) est daté de 2002. Le blog peut appartenir à ce que Marcoccia appelle la « communication numérique écrite » qui rassemble des productions écrites via internet ou bien le téléphone :

Les dispositifs de communication numérique écrites les plus connus sont le courrier électronique, le forum de discussion, la liste de diffusion, le tchat, la messagerie instantanée, le SMS (ou texto), le blog, les plateformes de réseaux sociaux. Le plus souvent l'expression de " communication numérique écrite » (ou " communication écrite en ligne ») réfère à des situations de communication interpersonnelle et n'intègrent pas les médias numériques de masse, comme le Web (dans sa version 1.0.), qui relève plus d'une problématique de publication que de communication. (Marcoccia 2016, introduction).

Le blog possède des caractéristiques techniques spécifiques : les billets sont présentés par ordre anté-chronologique (une certaine périodicité est attendue), des liens hypertextuels peuvent être utilisés (pour ouvrir le texte vers d'autres billets ou sur des textes extérieurs au blog. La première page du blog Les poissons n'existent pas présente donc le dernier billet de 2019 intitulé « La science des rennes de Noël », le classement anté-chronologique apparaît sous forme de colonne à droite et le texte en bleu, dans le $2^{\mathrm{d}}$ paragraphe, permet d'identifier un lien hypertexte qui renvoie à une photo :

Ex. 1 : extrait du blog Les poissons n'existent pas, http://fish-dont-exist.blogspot.com/2019/

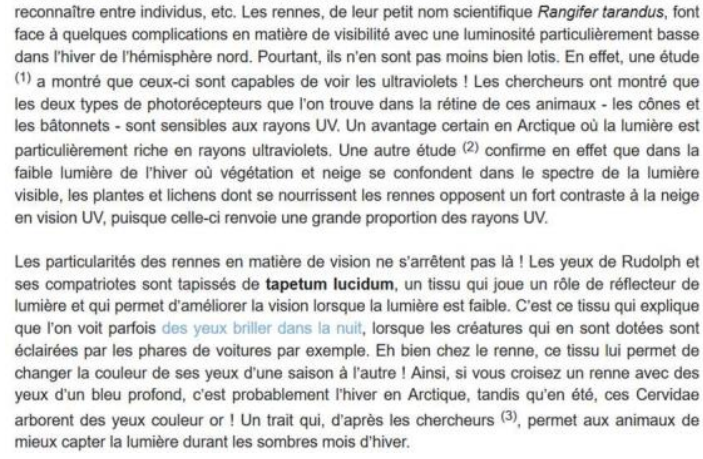


Les lecteurs de ce billet peuvent prendre le rôle de scripteurs en laissant des commentaires ; ils contribuent ainsi à la construction d'échanges avec l'auteur du blog mais aussi à des échanges entre les lecteurs/scripteurs eux-mêmes. Les caractéristiques technologiques permettent de nouvelles modalités d'écriture et de lectures ainsi qu'une nouvelle forme d'interactivité qui, au-delà du dialogue entre l'auteur du blog et l'ordinateur, donne naissance à un plurilogue qui se forme avec l'auteur et les lecteurs/scripteurs via le clavier et l'écran. Cette dénomination nouvelle de blog ainsi que les caractéristiques retenues participent à la détermination externe du genre.

On peut aussi s'interroger sur la dénomination même de blog scientifique. En effet, les créateurs de blogs autour de la science ont des profils très diversifiés : chercheurs, documentalistes, ingénieurs, journalistes scientifiques, étudiants, passionnés de science (Mounier, 2009). Les scientifiques y trouvent un support pour la diffusion de leurs travaux. Ainsi, si l'on s'intéresse à des critères macro-structurels, faudrait-il parler de blogs de scientifiques quand les auteurs diffusent leur domaine de recherche? Mais si l'on se focalise sur le destinataire, tous les blogueurs orientent leur propos vers un public moins spécialisé et de ce fait utilisent les procédés du discours de la vulgarisation scientifique. La délimitation entre blogs scientifiques et blogs de vulgarisation scientifique reste flottante, c'est pourquoi, nous proposons de concevoir la transmission de la science en terme de degrés ; en effet, dès qu'un scientifique s'exprime pour un public moins spécialisé, il est déjà du côté de la vulgarisation scientifique.

Comme «La richesse et la variété des genres de discours sont infinies car la variété virtuelle de l'activité humaine est inépuisable et [que] chaque sphère de cette activité comporte un répertoire des genres du discours qui va se différenciant et s'amplifiant au fur et à mesure que se développe et se complexifie la sphère donnée » (Bakhtine 1984, 265), il est intéressant de s'interroger sur la naissance possible d'un nouveau genre pour le blog de vulgarisation scientifique :

Du côté des chercheurs, qui doivent reconnaître la pertinence [des] catégories de sens commun, le genre est plutôt la représentation abstraite qu'ils élaborent à partir d'une collection de textes dotée d'une valeur sociale. Ainsi, en littérature, le critique évalue les textes réels à partir d'un texte parangon ; dans la vie professionnelle, on tend à fixer un modèle qui permet d'atteindre correctement un but. Dans les deux cas, les textes sont comparés pour être soit assimilés à une même catégorie, soit au contraire écartés comme trop éloignés du prototype de référence. (Branca-Rosoff 2007, p. 131).

Ainsi, il serait possible de considérer la vulgarisation scientifique comme un type de discours (Adam, 2001) et le blog de vulgarisation scientifique comme un genre, souvent porteurs de séquences explicatives : « [ce serait] reconnaître qu'il s'agit d'une pratique sociale établie qui répond à des normes stabilisées », comme pour le chat analysé par Branca-Rosoff (2007, p. 132). En nous appuyant sur Malrieu D. et Rastier F., 2001, nous proposons le schéma suivant :

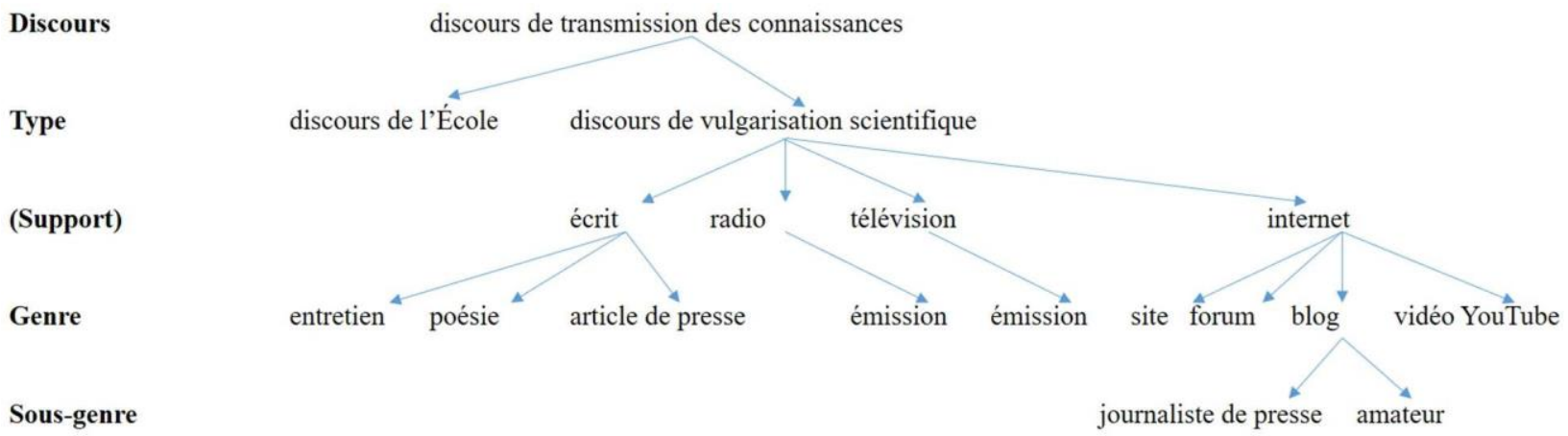

Le blog de vulgarisation scientifique emprunte des formes technologiques nouvelles proposées par le support internet et donc le medium est à prendre en considération pour l'identification d'un genre et sur le fond, il se présente comme un espace de transmission et de discussions autour de la science :

Les blogueurs de science utilisent les blogs comme des espaces ouverts de discussion. Ils reviennent ainsi à l'un des fondements essentiels de la démarche scientifique. Explication : lorsqu'un chercheur participe à un séminaire, ce qu'il échange est considéré comme transitoire. Mais s'il publie ses idées sur Internet, un autre chercheur peut, même plusieurs mois après, poser son commentaire, le rapprocher de ses travaux, continuer à échanger. «On touche à une dimension heuristique, propre à la découverte, qui correspond à une pratique très ancienne, la disputatio, ou tout simplement l'échange de discussions entre savants », résume Marin Dacos, directeur du CLEO, Centre pour l'édition électronique ouverte. (Cité des sciences et de l'industrie, le Blob, l'extra-média, https://leblob.fr/).

On constate ici la nécessité de prendre en compte le rôle actif du chercheur dans la construction des catégories de genres (Branca-Rosoff 2007, p. 132). C'est pourquoi, nous avons mis en évidence des critères macro-structurels et microstructurels pour caractériser le blog. Les critères micro-structurels (voir aussi l'introduction) se retrouvent dans le discours de vulgarisation quel que soit le genre et l'aire culturelle (voir partie 2). Pour l'exemple 2, issu d'un blog québécois, on relève une définition de terme spécialisé, Le système vestibulaire est un organe sensoriel situé dans l'oreille, une définition proposées en note avec « otolithes », une reformulation : cellules ciliées - des cils rattachés directement aux neurones - qui regroupent des éléments co-référentiels ainsi que l'exploration de la nomination avec le verbe « appeler » : 
des cristaux appelés « otolithes »* :

Ex. 2 : extrait du blog Dire, Agence Science-Presse, 4/12/2019,

https://www.sciencepresse.qc.ca/blogue/dire/2019/12/04/equilibre-renversant

\section{Le vertige}

Le système vestibulaire est un organe sensoriel situé dans l'oreille. Son anatomie est complexe : on parle de « labyrinthe », car il est constitué d'un ensemble de cavités osseuses. Celui-ci est sensible aux accélérations angulaires, soit les rotations de la tête dans les trois plans de l'espace et les déplacements linéaires, dans les plans horizontal et vertical. C'est ce qui permet à Émilie, comme à tout être humain, de savoir si l'ascenseur monte ou descend lorsqu'elle se trouve à l'intérieur de la cabine sans fenêtre. Les structures du système vestibulaire sont garnies de cellules ciliées - des cils rattachés directement aux neurones - baignant dans une substance gélatineuse qui contient à sa surface des cristaux appelés « otolithes »*.

Lexique

Otolithes : petits cristaux de carbonate de calcium qui sont situés sur une membrane gélatineuse dans l'oreille interne et qui, lors d'un mouvement de la tête, font «glisser » la membrane reliée aux cellules ciliées d'un bord ou de l'autre grâce à leur poids sous la gravité.

Ces marques linguistiques sont caractéristiques du discours de vulgarisation scientifique. Afin de mettre à l'épreuve ces caractéristiques, nous avons engagé une collaboration sur la comparaison des discours (Grillo V. de C. S., Reboul-Touré S. et Glushkova M., éds, à paraître) et grâce à ces collaborations avec des chercheurs du groupe Diálogo - autour de Grillo V. de C. S., à l'université de São Paulo - nous analysons les discours de vulgarisation scientifique au travers de blogs en France et au Brésil (Machado à paraître). Cette collaboration nous a permis d'explorer une nouvelle catégorie d'analyse notamment celle des « sphères d'activité langagière » (voir 4. ci-dessous)

\section{La comparaison : des aires discursives et culturelles différentes}

La comparaison est ainsi définie dans le Trésor de la langue française : « acte intellectuel consistant à rapprocher deux ou plusieurs animés, inanimés concrets ou abstraits de même nature pour mettre en évidence leurs ressemblances et leurs différences $»$. Cette définition va de soi et elle semble très ordinaire mais elle est à spécifier quand on utilise la notion dans un domaine particulier, notamment celui des sciences du langage. C'est plutôt du côté de la sociolinguistique que l'on trouve quelques éléments de réflexion autour de la comparaison : « Une bonne part du travail en (socio)linguistique consistant à comparer (des phénomènes, des structures, des énoncés, des événements discursifs et [...] des corpus ou des parties de corpus), la comparabilité mérite d'être considérée en soi, au-delà des évidences ». (Gadet et Wachs, 2015) ; ces linguistes proposent d'étudier la propriété de comparabilité qui prend en compte des " paramètres formalisables, quantifiables, en dichotomie (le sexe) ou par strates (l'âge ou la classe sociale) » par exemple (Reboul-Touré, à paraitre b). La comparaison, dans le domaine de l'analyse du discours, pourra être considérée comme une méthode heuristique avec une analyse comparative entre des corpus qui n'avaient pas encore été mis en regard car appartenant à des aires culturelles différentes. « Comparer, c'est en effet accepter non seulement de se décentrer, mais également rendre plus exigeants la formulation d'hypothèse et le travail de théorisation » (Vigour, 2016) (idem). Comparons quelques éléments des blogs en France et au Québec.

En France, le premier texte qui diffuse la science, et plus particulièrement l'astronomie, est conçu par Fontenelle. Les Entretiens sur la pluralité des mondes, 1686 peut être considéré comme le premier texte qui transmet des connaissances liées à l'astronomie pour un public moins savant dans le domaine comme le montre certains aspects de la préface :

« Je dois avertir ceux qui liront ce livre, et qui ont quelque connaissance de la physique, que je n'ai point du tout prétendu les instruire, mais seulement les divertir en leur présentant d'une manière un peu plus agréable et plus égayée ce qu'ils savent déjà plus solidement ; et j'avertis ceux pour qui ces matières sont nouvelles que j'ai cru pouvoir les instruire et les divertir tout ensemble ».

On ne parle pas de vulgarisation scientifique à l'époque mais cette catégorisation sera proposée dans le cadre d'une analyse linguistique, celle de M.-F. Mortureux qui a élaboré sa thèse d'état sur l'étude de la vulgarisation scientifique dans l'œuvre de Fontenelle. Lors des siècles suivants, les auteurs diffusent la science via des genres littéraires et notamment la poésie puis ils se détachent de ces genres :

La fin de l'Ancien régime et l'Empire marquent l'apogée d'une poésie scientifique en vers qui perd son prestige avec le triomphe du Romantisme, avant d'entamer un lent déclin, jusqu'à la disparition du genre, au début du vingtième siècle. Tout se passe comme si les critiques qui reprochaient de longue date au vers une incapacité à transmettre correctement la science, pour n'offrir, selon le mot de Buffon, qu'une parole où « la raison porte des fers », obtenaient gain de cause. (H. Marchal, 2008).

C'est à la fin du XIX ${ }^{\mathrm{e}}$ siècle qu'apparaissent des revues de vulgarisation scientifique : La Nature, Revue des sciences et de leurs applications aux arts et à l'industrie, 1873 ; Eurêka. Tribune des inventeurs. Revue illustrée de vulgarisation scientifique et industrielle, 1892 ; L'étincelle électrique, 1880 ; L'ingénieur civil. Journal d'application et de vulgarisation des découvertes les plus récentes, 1892 ; Revue photographique de l'Ouest, 1906. Aujourd'hui, les magazines sont les suivants : Ça m'intéresse, premier numéro en 1981 ; Ciel et espace, 1945 ; Cosinus, 1999 ; Pour la science, 1977 ; La recherche, [1946], 1970 ; Science et Avenir, 1947 ; Science et vie, 1913 ; Science et vie junior, 1988. 
De nouvelles créations sont proposées dans les années 2000, avec une diversité au niveau des thématiques ou bien des publics : Science et vie découvertes, 2000 ; Cerveau et psycho, 2003 (Reboul-Touré 2012) et la dernière création sortie à l'automne 2018, le magazine Curionautes des sciences pour les enfants dès 8 ans. Ce rapide parcours historique montre une tradition bien installée en France pour la vulgarisation de la science (depuis le XVIIe siècle) et souligne l'existence de recherches en analyse du discours depuis les années quatre-vingt sur ce type de discours (Mortureux, 1982, Beacco, Moirand 1995).

Au Québec, même si le français est très présent, culturellement l'influence de l'anglais est forte, ce qui a des retombées aussi dans les choix de politique de vulgarisation scientifique :

Alors qu'en Amérique du Nord le soutien aux initiatives tient lieu de politique, les pays européens développent des politiques scientifiques plus élaborées. Inspiré par ces deux cultures, le Québec se trouve à mi-chemin des deux approches, l'une institutionnalisée et la seconde davantage orientée vers le soutien aux initiatives. La province a ainsi développé ses propres organismes, évènements et émissions de vulgarisation scientifique en français. (Bryère et Bricaud, 2016, p. 1)

Il va de soi qu'une coopération en analyse du discours sur la vulgarisation scientifique est à mettre en place pour une meilleure compréhension des phénomènes culturels. Cependant, des documents administratifs nous permettent de cerner des choix politiques du côté du Québec pour la période actuelle :

Les journaux quotidiens proposent des articles d'actualité scientifique sur leur site internet, dont le contenu peut être parfois payant. Certains de ces journaux et autres médias disposent d'une rubrique «science » (La Presse, Radio-Canada, Le Devoir, Le journal de Montréal) ou de rubriques thématiques «Environnement », "Santé » (Le Devoir)... De nombreux journalistes scientifiques possèdent également leur propre blog, qu'il soit lié à leur journal ou indépendant (Jean-François Cliche pour La Presse, Valérie Borde pour l'Actualité...). Le site web de l'Agence Science-presse propose une rubrique Science! on blogue, agrégeant des articles de blogs québécois. (Bryère et Bricaud, 2016, p. 7)

Une des spécificités du Québec est de fortement promouvoir la recherche et la vulgarisation en français :

L'Acfas ${ }^{5}$ défend une science qui se fait et se communique en français. La défense de la francophonie au Québec est un axe majeur de la politique du gouvernement provincial, dans un pays où la majorité de la population est anglophone. À ce titre, il n'existe pas d'équivalent de l'Acfas en France. (idem, p.11)

Cet angle pour une comparaison discursive permet de noter que les choix politiques vis-à-vis de la vulgarisation de la science suivent des orientations différentes. Au Québec, pour les sites du portail retenu, ce sont essentiellement des scientifiques ou des journalistes qui créent des blogs alors qu'en France, on trouve certes ces catégories de blogueurs mais on relève un contraste intéressant car au-delà de ces intervenants, on identifie une nouvelle figure, notamment celle de l'amateur parmi les créateurs de blogs.

Un autre espace de l'analyse du discours liée à la comparaison des langues et des cultures s'est mis en place avec l'innovation portée par les chercheurs du Cediscor (Cislaru, 2006 ; Claudel et alii (éds) 2013, von Münchow 2004, 2011, 2013) qui se sont consacrés au développement de ce que von Müchow appelle aujourd'hui l'analyse du discours contrastive (ADC), pour comparer, contraster des langues, des cultures distinctes (dans Grillo V. de C. S, et alii, éds, à paraître), sont présentés des analyses entre français/japonais, allemand/français, français/portugais, portugais/russe, à travers des concepts d'analyse du discours, de linguistique textuelle et des théories de l'énonciation. On peut relever le concept de tertium comparationis, formulé par ces chercheurs, concept qui a été utile pour effectuer des comparaisons réalisées par ceux du groupe Diálogo (CNPq/USP) (Grillo et Glushkova, 2016 ; Grillo et Higachi, 2017). Le trait caractéristique du collectif brésilien est de développer une analyse comparative basée sur les concepts et les procédures méthodologiques de Bakhtine et de son Cercle. Les résultats de ces échanges scientifiques, notamment au sujet des blogs de vulgarisation scientifique au Brésil et en France apparaissent dans les analyses ci-dessous. 


\section{Les figures de la vulgarisation scientifique}

Les travaux de recherche dans les années 1980-90 mettent en évidence une configuration typique de la vulgarisation scientifique avec 3 pôles : le scientifique, le vulgarisateur et le public avec des positions descendantes, le scientifique ayant le savoir est dans une position haute. Jacobi parle alors de la place du $3^{\mathrm{e}}$ homme pour le vulgarisateur :

L'art du journaliste vulgarisateur consisterait d'une part à retrouver la nudité de la vérité scientifique et à la traduire dans une langue, plus simple, à la portée de tout un chacun. En fait une telle opinion n'est propre ni au lecteur moyen, ni au vulgarisateur. Les scientifiques eux-mêmes sont très critiques vis-à-vis du jargon des sciences... autre que le leur et le mépris dans lequel des spécialistes des sciences « dures » tiennent les terminologies de la linguistique ou de la sociologie est immense. (Jacobi, 1985).

$\mathrm{Ce}$ « troisième homme » présent dans les articles des revues de vulgarisation scientifique (sous forme papier) peut aujourd'hui tenir un blog, comme Sylvestre Huet pour le journal Le Monde :

Ex. 3 : extrait du blog Science ${ }^{2}$, https://www.lemonde.fr/blog/huet/

\section{$\left\{\right.$ Sciences $\left.^{2}\right\}$}

Le blog de Sylvestre Huet, journalist
spécialisé en sciences depuis 1986

15 DECEMBRE 2019

Musées de sciences contre la science?

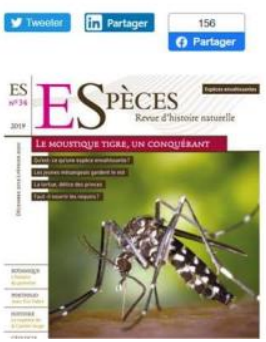

ARTICLES RECENTS

Musées de sciences contre la science ?

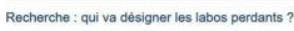

Antoine Pelti contesté au CNRS

Le dernier numéro de la formidable est question d'espéces

moustique tigre present désormais

dans la majorité des départements

français. De lhistoire du pommier -
de son origine sauvage dans les

montagnes du Kazakhstan a ses
montigine sauvage dans les

avatars domestiques, issus de

par Thomme afin d'obtenir les
meilleures pommes. Du savoureux

Climat : une mauvaise nouvelle de 6000 ans

43,1 gigatonnes de $\mathrm{CO} 2$ émis en 2019

COMMENTAIRES RECENTS

timiota dans Un séisme majeur menace Istanbul

Desman dans Un seitsme majeur menace Istanbu

Sylvestre Huet se présente comme un journaliste spécialisé en sciences depuis 1986 et il intervient régulièrement sur son blog Sciences $^{2}$. Les articles sont liés à l'actualité et présentent souvent une articulation entre science et politique.

Autre exemple, le journal Le Monde présente aussi un blog, Passeur de sciences :

Ex. 4 : extrait du blog Passeur de sciences, https://www.lemonde.fr/passeurdesciences/

\section{SCIENCES - PASSEUR DE SGEENCES \\ BiLLet de BLOG \\ Découverte de tombes de grands \\ 2. Pierre Barthelemy prêtres égyptiens et autres infos scientifiques}

Au menu de la 380 e sélection hebdomadaire : ce que Donald Trump pense du changement climatique, des aurores boréales en forme de dunes, comment pirater une voiture...

Publie lo 31 janvior 2020 a 18 h29 i O Lecture 1 min.

"Créé en 2011, Passeur de sciences raconte les petites et grandes nouvelles dans l'actualité des sciences et de l'environnement. Ce blog est animé par Pierre Barthélémy, journaliste scientifique au Monde ». Le choix fait par le journaliste est ici différent : son blog se présente comme une revue de presse d'articles scientifiques, chaque annonce renvoie via un lien hypertextuel à un article issu d'autres sources :

Ex. 5 : idem 


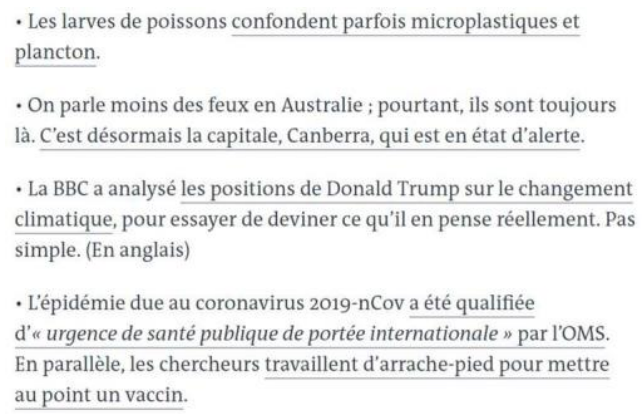

La vulgarisation est parfois réalisée directement par des scientifiques : certains d'entre eux avaient osé cette diffusion sur les supports papier, à la radio et à la télévision pour devenir des vulgarisateurs renommés comme par exemple, Hubert Reeves. Les scientifiques s'emparent volontiers des formes présentes sur internet et ils sont nombreux à vulgariser directement (sans l'intermédiaire du « troisième homme ») leurs connaissances. Voici, par exemple, le blog de Bernard Valeur :

Ex. 6 : extrait $\mathrm{du}$ blog Questions de couleurs, 03/01/2020 http://www.scilogs.fr/questions-decouleurs/author/bernardvaleur/

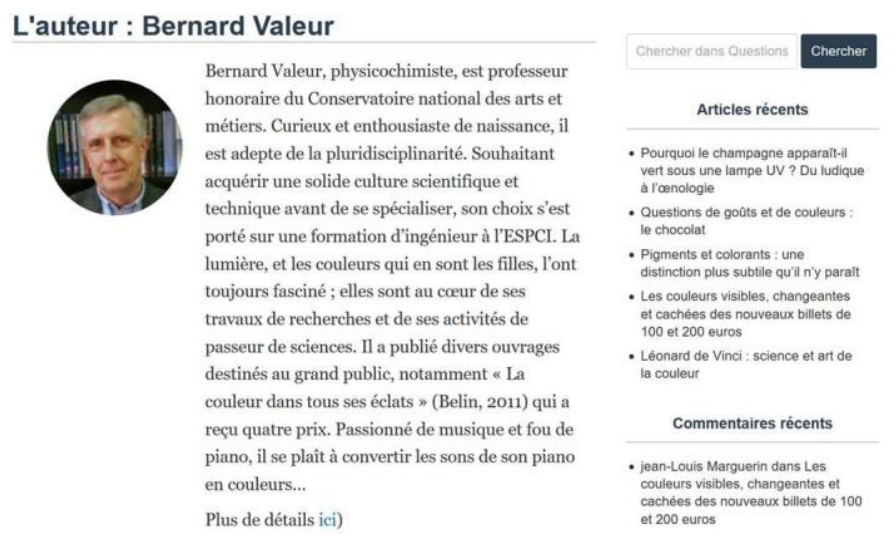

Il se présente comme physicochimiste, est professeur honoraire du Conservatoire national des arts et métiers et fait partie des figures des scientifiques qui transmettent les connaissances via des blogs.

C'est en diversifiant les corpus et en analysant les discours de la science dans la presse quotidienne, un lieu qui était orienté plutôt du côté de l'actualité, que nous avons trouvé des traces de vulgarisation scientifique lorsque l'actualité s'y prêtait : autour des événements comme les tremblements de terre, par exemple 6 . Puis, la convergence entre science, médias et société est devenue prégnante plus particulièrement autour des « affaires sanitaires » (la vache folle, le H1N1, la grippe porcine, la grippe aviaire...) et dans ces discours des marques de vulgarisation étaient identifiables de manière parcellaire. La nouveauté portée par ces corpus est apparue, entre autres, autour des intervenants avec de nouvelles figures : celles de l'expert ou du citoyen qui sont entrées linguistiquement dans les discours au travers du discours rapporté sous toutes ses formes (alors qu'auparavant, ces intervenant n'étaient pas entendus dans ce contexte) ${ }^{7}$.

Avec l'analyse des blogs de vulgarisation scientifique, en France, une nouvelle configuration se fait jour avec l'apparition d'une nouvelle figure, celle de l'amateur. En effet, le journaliste scientifique bien identifié au niveau institutionnel comme le passeur de la science pour un plus grand public demeure présent dans les revues de vulgarisation qui sont clairement identifiées comme des transmetteurs de connaissances. Certains journalistes vont tenir des blogs mais tou.te.s les bloggueur.e.s n'ont pas ce statut. Ces nouveaux intervenants contribuent à la «montée en puissance des amateurs » (Rieffel, 2014) qui participent au mouvement d'élargissement des savoirs et des compétences relevant d'une sorte d'autoapprentissage puisque contrairement aux hiérarchies scolaires aucun diplôme n'est exigé :

On découvre ainsi un monde pour le moins complexe, forgé selon des modalités inédites d'apprentissage des connaissances, constitué d'échanges en réseau au sein de communautés virtuelles, de procédures d'hybridation et de braconnage très variées qui soit ébranlent les processus traditionnels d'acquisition des savoirs, soit se situent carrément à côté ou en marge des activités traditionnellement les plus légitimes. Nous sommes ici typiquement dans le cadre de l'innovation ascendante, venue du peuple et non plus des élites, célébrées par tous les fondateurs du web. (Rieffel, 2014, 128) 
Les internautes amateurs se présentent comme de simples blogueurs, comme le montre l'auteur de Couleur de science :

Ex. 7 : extrait de Couleur Science, 8/11/2019, https://fr.tipeee.com/couleur-science

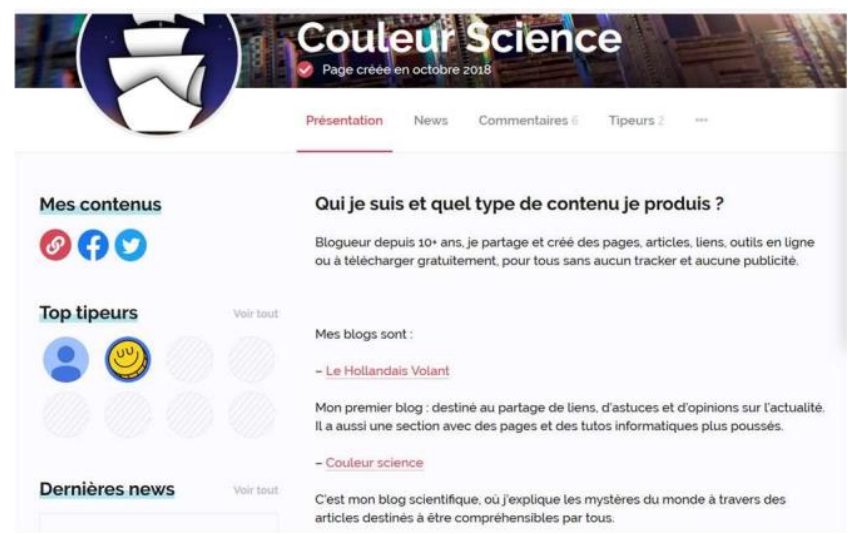

Rieffel, professeur en sciences de l'information et de la communication, analyse ce phénomène comme étant de l'ordre de la « désintermédiation » :

Les nouvelles technologies numériques engendrent à des degrés divers, une perte des repères traditionnels en matière culturelle qui conforte l'émergence d'un phénomène majeur : la fin (ou du moins la diminution de l'influence) des médiateurs et des intermédiaires (la "désintermédiation" dans le jargon des spécialistes). [...] à partir du moment où tout le monde peut a priori expérimenter et créer grâce aux nouveaux supports et outils numériques [...], le rôle des professionnels et des experts perd de son importance. [...] Or peut-on véritablement imaginer une démocratie sans corps intermédiaires, peut-on transmettre une culture sans contextualisation et sans véritable hiérarchisation? (Rieffel 2014, p. 265-266)

Les commentaires font se rencontrer des internautes sur des thématiques scientifiques et face à ces nouveaux regroupements d'internautes autour de la vulgarisation scientifique, on peut voir émerger de nouvelles communautés. C'est bien le type de discours qui rassemble ces différentes figures (journaliste scientifique, scientifique, amateur) dont les discours partagent des caractéristiques linguistiques similaires. Mes prochaines recherches porteront sur les différences qu'il peut y avoir entre les blogs selon le profil des énonciateurs ; une première caractéristique peut déjà être isolée, l'humour qui peut être présent chez les bloggueurs amateurs. Par exemple, l'auteur du blog Sauvages du Poitou se présente ainsi : «Sauvages du Poitou, c'est de la botanique joyeuse, des herbes folles et des bestioles plein le jardin ! Derrière le clavier, l'appareil photo et les crayons de couleur : Norb, apprenti botaniste, apprenti dessinateur et apprenti shérif à la poursuite des mauvaises herbes et hors-la-loi dont les fleurs sont mises à prix » et voici le titre d'un de ses billets :

Ex. 8 : extrait de Sauvages du Poitou, https://www.sauvagesdupoitou.com/rech/héron

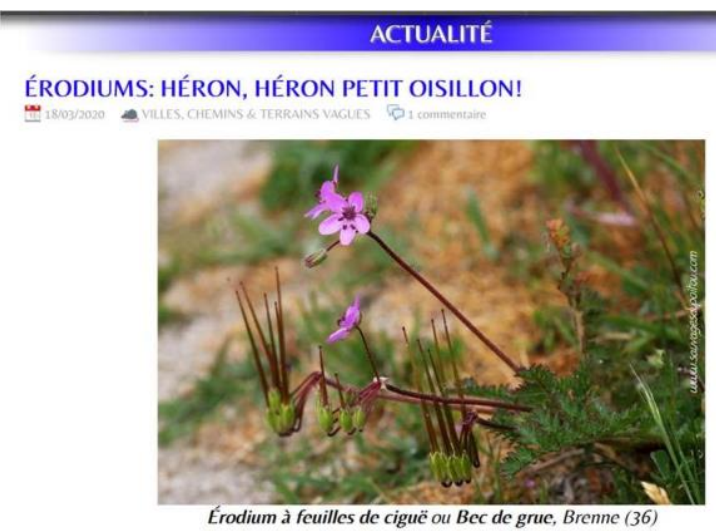

«Érodiums : héron, héron petit oisillon » qui fait écho à une chanson populaire Il était une bergère dont le refrain est : «Et ron et ron, petit patapon ». Les érodiums rassemblent des plantes dont certaines sont appelées becs-de-grue or l'étymologie d'érodium est plutôt héron. Ce clin d'œil aux oiseaux se retrouve aussi dans le billet : « J'en vois certains qui baillent aux corneilles devant autant de gros mots ». Ce trait d'humour pourrait marquer certains blogs d'amateur et en faire un « sous-genre » (?). 


\section{Vers des sphères d'activités langagières}

Les blogs de vulgarisation scientifique rassemblent des auteur.e.s qui jusqu'alors n'avaient pas eu l'occasion de mettre en avant leurs points communs : un intérêt pour la vulgarisation de la science et le partage de leur recherche et/ou passion via une écriture ouverte passant par internet, sous la forme du blog. L'analyse du discours des blogs de vulgarisation scientifique peut emprunter le versant de «l'ethnologisation » qu'elle a rencontré dans les années 1990 :

L'intérêt pour les discours scientifiques n'est pas nouveau. Mais jusqu'à une date récente, c'était plutôt l'affaire de la sociologie de la science que de l'analyse textuelle. Dès les années 60 , on n'avait pas hésité à utiliser des problématiques ethnologiques pour appréhender le fonctionnement des communautés scientifiques et le rôle qu'y joue la production des textes dont il n'est pas difficile de mettre en évidence les enjeux sociaux (ou plutôt « microsociaux »). L' « ethnologisation » de l'analyse du discours permet de faire le chemin en sens inverse : partant d'un fonctionnement textuel, on cherche à mettre au jour le réseau de transactions sociales que celui-ci implique, en s'efforçant de ne pas juxtaposer les deux instances. En effet, l'important est de nouer pratique textuelle et pratique institutionnelle : si d'une certaine façon l'énonciation constitue une institution discursive, en retour l'institution n'est pas pensable indépendamment de l'exercice de la parole qu'elle prescrit. C'est dire que le texte n'est pas un simple support d'informations, qu'il a une économie propre, laquelle n'est pas intégralement linguistique. (Maingueneau, 1992, 120)

Ces éléments d'analyse sont transposables au fonctionnement des communautés liées à la «fabrication » de la vulgarisation scientifique et c'est bien à partir de caractéristiques linguistiques (hétérogénéité discursive, reformulation, etc.) liées au fonctionnement textuel que l'analyste du discours cherche à identifier où émergent ces « transactions sociales ». C'est sur cet aspect de l'analyse qu'il nous paraît intéressant de passer de la notion de communautés discursives à celle de "sphères d'activité langagière » :

On peut alors faire l'hypothèse qu'à [d]es sphères d'activité humaine correspondent des sphères d'activité langagière, assez proches de ce que l'analyse du discours française appelle des « formations discursives » [...] On peut ainsi postuler qu'à une sphère d'activité humaine correspondent plusieurs "sphères d'activité (s) langagière (s) » et que dans le domaine de la diffusion des sciences et des techniques différentes sphères d'activité langagière sont inévitablement conduites à se rencontrer ${ }^{8}$. (Moirand S., Reboul-Touré S., Pordeus M., 2016, 146)

Les sphères d'activité langagière autour des blogs de vulgarisation scientifique rassemblent autour d'un blogueur tous les lecteurs/scripteurs qui peuvent jouer un rôle plus ou moins grand dans la construction de la transmission des connaissances.

L'analyse des blogs de vulgarisation scientifique permet aussi de faire apparaître des interactions instantanées ou différées qui pourraient s'apparenter au courrier des lecteurs mais avec une technologie qui facilite et même sollicite des commentaires :

Ex. 6 : un commentaire suite à un billet

Damien 12.09.2013 15:21 Répondre | Permalink

Bonjour et merci pour cet article qui nous donne une vision à contre courant sur ses termes de vulgarisation.

Malgré le fait que l'on retrouve dans une multitude de livres écrits par des scientifiques respectés dont le titre emploi les mots d'infiniment grand ou petit, je porte un grand crédit a votre façon de penser.

Une question me trotte dans la tête suite à cette lecture, quels mots emploieriez-vous en lieu et place de "infiniment grand" et "infiniment petit" dans un livre de vulgarisation? (Reboul-Touré 2015)

La justesse des propos présents sur les blogs est régulée au niveau des commentaires car les internautes peuvent relever des erreurs. On constate ainsi une collaboration positive pour apporter un degré de qualité ; les commentaires peuvent participer à la précision dans la transmission des connaissances :

Ex. 7 : extrait de Quoi dans mon assiette ?, 03/12/2019, https://quoidansmonassiette.fr/quels-sont-les-dangers-descomplements-alimentaires-et-risques-pour-la-sante/ 


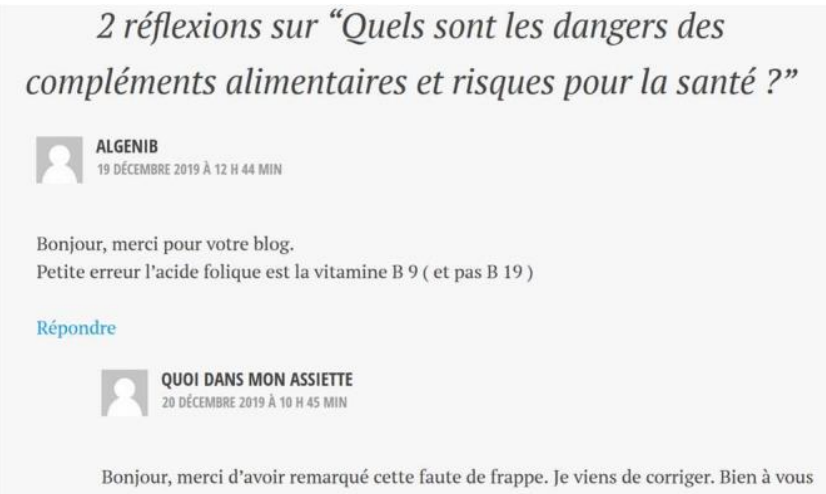

Par ailleurs, les commentaires étant visibles par tous, il peut y avoir des commentaires sur les commentaires, et donc le statut d'internaute permet à un grand nombre de personnes qui étaient jusqu'alors silencieuses de prendre la parole sans intermédiaire. La transmission descendante des connaissances par l'intermédiaire du vulgarisateur cohabite avec un autre circuit : une transmission horizontale des connaissances entre internautes.

Suite à des remarques, l'auteur du blog peut ajuster son discours. Les lecteurs/scripteurs jouent un rôle actif dans la transmission des connaissances sur internet, notamment au travers des blogs (les commentaires se développent aussi sur des sites, suite à des vidéos sur youtube ainsi que sur les réseaux sociaux comme twitter) :

Les quidams ont conquis Internet. [...] En France, Wikipédia réunit un million d'articles, et dix millions de blogs ont été créés. Un quart des internautes a déjà signé une pétition en ligne. Ces quelques chiffres illustrent un phénomène essentiel : le web contemporain est devenu le monde des amateurs. [...]

De même que nous avons vécu depuis deux siècles une double démocratisation, à la fois politique et scolaire, de même nous entrons dans une nouvelle ère de démocratisation, celle des compétences. (Flechy 2014, p.7)

La prise en considération des lecteurs peut même devenir le moteur pour cibler certains aspects de la vulgarisation scientifique au sein de sphère d'activité langagière comme nous pouvons le voir avec un blog de vulgarisation scientifique qui s'adresse à des enfants, Kidi 'science, depuis 2012 (sur le portail de Café des sciences) :

Ex. 8 : extrait de Kidi'science, https://kidiscience.cafe-sciences.org/articles/bilan-2019-et-projets-pour-2020/

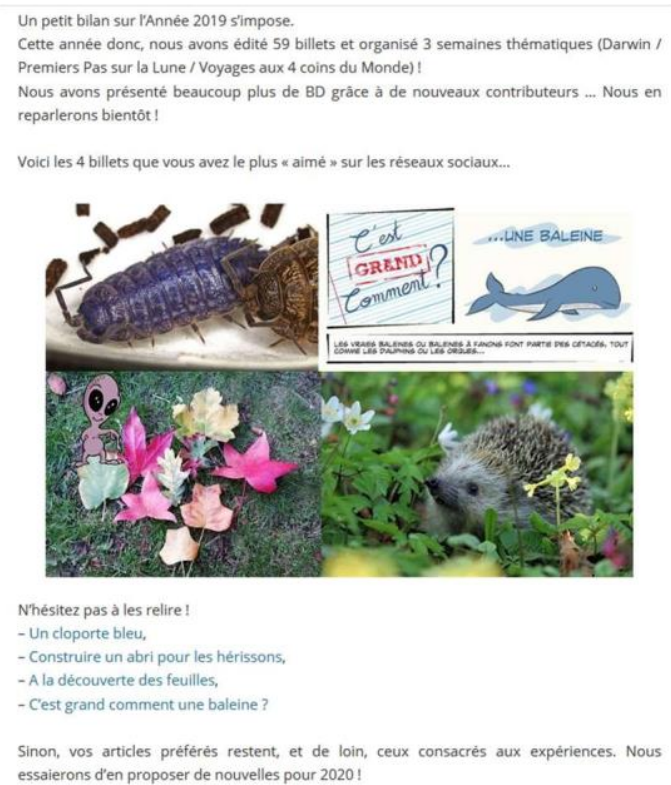

Les auteurs de ce blog précisent en effet que suite aux commentaires reçus, ils développeront les articles consacrés aux expériences. Les interactions auteurs-lecteurs/scripteurs sont importantes au sein de la sphère d'activité langagière. 


\section{Pour conclure}

La vulgarisation scientifique depuis les Entretiens sur la pluralité des mondes de Fontenelle s'est glissée dans différents genres, entretien, poésie, roman, bande-dessinée, article de revues, article de journal pour l'écrit mais aussi via des médias comme la télévision ou la radio. Le support internet a relancé la circulation des discours de vulgarisation scientifique, notamment au travers des sites ouverts par les revues papier comme La Recherche ou Science et vie mais aussi de sites spécifiquement conçus pour le support internet comme Futura Sciences, en France. Les blogs, qui au début de leur création s'approchent plus du journal intime ou du carnet de bord, semblent une nouvelle forme des pages personnelles (Beaudouin, Fleury et Pasquier 2004). Le blog de vulgarisation scientifique, offrant des facilités de publication et d'interaction, rencontre un grand succès. Au sein de la «communication numérique écrite », il peut être considéré comme un genre.

Nous avons ici rassemblé des catégories qui peuvent entrer dans un continuum entre analyse intralinguistique et linguistique du discours, comme le proposent Adam, Grize et Ali Bouacha (2004) :

\section{Linguistique du discours}

\begin{tabular}{|c|c|c|}
\hline $\begin{array}{l}\text { Linguistique } \\
\text { du systène } \\
\text { (« sémiotique } »)\end{array}$ & $\begin{array}{l}\text { Linguistique de } \\
\text { l'énonciation } \\
\text { (" sémantique ") }\end{array}$ & $\begin{array}{c}\text { Translinguistique } \\
\text { des textes, } \\
\text { des ceuvres }\end{array}$ \\
\hline
\end{tabular}

\section{Analyse intralinguistique}

La reformulation peut se situer entre intralinguistique et linguistique de l'énonciation, les hétérogénéités énonciatives, du côté de la linguistique de l'énonciation et les sphères d'activité langagière, du côté de la linguistique du discours.

La recherche autour de la comparaison et la collaboration avec des chercheur.e.s du groupe Diálogo m'ont permis de confirmer l'intérêt de nouvelles catégories pour l'analyse de la vulgarisation scientifique, entre autres, la figure de l'amateur et les sphères d'activités langagières. Ces catégories identifiables à partir des corpus retenus sont sans doute transposables à l'analyse des discours d'autres blogs, même ayant des thématiques différentes car il s'agit de catégories macro-structurelles.

La vulgarisation scientifique, grâce à ce support, connaît un nouvel essor inattendu auprès des internautes créant ainsi une forme hybride de discours de transmission des connaissances. En effet, l'analyse de ces discours montrent que des catégories déjà identifiées sur le support papier (voir introduction) sont présentes et que l'aspect technologique permet à l'interaction de se mettre en place avec des échanges via ordinateur présents dans les commentaires et aussi par l'utilisation des liens hypertextes qui offrent des parcours de lectures infinis : chaque lecteur peut ouvrir un lien ou non et donc en créant son propre itinéraire, il va chercher les éléments de connaissances qui lui convient. Ce discours tentaculaire, renouvelé à chaque lecture pourrait constituer un hyperdiscours.

\section{Références bibliographiques}

Ablali, D., Achard-Bayle, G., Reboul-Touré, S. et Temmar, M., éds. (2018). Texte et discours en confrontation dans l'espace européen . Berne : Peter Lang.

Adam, J.-M. (2001). Types de textes ou genres de discours? Comment classer les textes qui disent de et comment faire ?. Langages, 141, p. 10-27.

Adam, J.-M., Grize, J.-B. et Ali Bouacha, M., éds. (2004). Textes et discours : catégories pour l'analyse. Dijon : Éd. universitaires de Dijon.

Authier, J. (1982). La mise en scène de la communication dans des discours de vulgarisation scientifique. Langue française, 53, p. 34-47.

Authier-Revuz, J. (1984). Hétérogénéité(s) énonciative(s). Langages, 73, p. 98-111. www.persee.fr/doc/lgge_0458726x_1984_num_19 73 _1167

Bakhtine, M. (1984[1952-1953]). Esthétique de la création verbale. Paris : Gallimard.

Beacco, J.-C. et Moirand, S. (1995). Autour des discours de transmission de connaissances. Langages, 117, 32-53, http://www.persee.fr/doc/lgge 0458-726x 1995 num $29117 \quad 1704$

Beaudouin, V., Fleury, S. et Pasquier, M. (2004). Les pages personnelles comme terrain d'expérimentation. in Mourlhon-Dallies, F., Rakotonoelina, F. et Reboul-Touré, S., éds, Les Carnets du Cediscor 8 - Les discours de l'internet, PSN, 143-164.

Bouquet, S., Grillo, V. de C. S. (2007). Introduction. Linx 56 - Linguistique des genres, 56, p.7-18.

Branca-Rosoff, S. (2007). Genres et activité langagière : l'exemple des tchats. Linx, 56, 127-141. 
Bryère, P. et Bricaud, M. (2016). La culture scientifique au Québec, Consulat général de France à Québec

https://www.google.com/search?client=firefoxbd\&channel=crow\&q=bricaud+culture+scientifique+quebec

Cislaru, G. (2006). Noms de pays et autoreprésentation dans le discours des périodiques nationaux français, anglophones, roumanophones et russes. Les Carnets du Cediscor, 9, PSN, https://journals.openedition.org/cediscor/65

Claudel, C., von Münchow, P., Pordeus Ribeiro, M. Pugnière-Saavedra, F. et Tréguer-Felten, G., éds. (2013). Cultures, discours, langues : nouveaux abordages. Limoges : Lambert-Lucas,

Gadet, F. et Wachs, S. (2015). Comparer des données de corpus : évidence, illusion ou construction ?. Langage et société, 154, p. 33-49.

Grillo Vieira de Camargo, S. (2007). Epistémologie et genres du discours dans le cercle de Bakhtine. LINX, 56, 19-36.

Grillo V. de C., S. et Glushkova, M. (2016). A divulgação científica no Brasil e na Rússia: um ensaio de análise comparativa de discursos". [La vulgarisation scientique au Brésil et en Russie : un essai d'analyse comparative des discours], Bakhtiniana - revista de estudos do discurso, v. 11, p. 69-92.

Grillo V. de C., S. et Higachi, A. (2017). Enunciados verbo-visuais na divulgação científica no Brasil e na Rússia: as revistas Scientific American Brasil e v míre naúki (no mundo da ciência). [Des énoncés verbo-visuels dans la vulgarisation scientifique au Brésil et en Russie : les revues Scientific American brasil e V míre naúki (dans le monde de la science)] in Kozma, E. V. B. et Puzzo, M. B. (Org.). Múltiplas linguagens: discurso e efeito de sentido [Des langages multiples : discours et effet de sens]. 1ed.Campinas: Pontes, 2017, v. 1, p. 91-130.

Grillo V. de C., S., Reboul-Touré, S. et Glushkova, M., éds. (à paraître). Analyse du discours et comparaison : enjeux théoriques et méthodologiques.

Jacobi, D. (1985). Sémiotique du discours de vulgarisation scientifique. Semen, 2, http://journals.openedition.org/semen/4291

Jurdant, B. ([1973], 2009). Les problèmes théoriques de la vulgarisation scientifique. Éditions des archives contemporaines, https://www.researchgate.net/publication/305389883 Les_problemes theoriques de la vulgarisation_scientifique

Le Cam, F. (2006). Etats-Unis : les weblogs d'actualité ravivent la question de l'identité journalistique. Réseaux, 138, p. 139-158.

Machado, F. S. (à paraître). Aspects de la divulgation scientifique dans les blogs brésiliens. in Grillo V. de C., S., Reboul-Touré, S. et Glushkova, M., éds.

Maingueneau, D. (1992). Le tour ethnolinguistique de l'analyse du discours. Langages, 105, p. 114-125.

Maingueneau, D. (2017). Quelques réflexions sur l'évolution de l'analyse du discours. in Ablali, D., Achard-Bayle, G., Reboul-Touré, S. et Temmar, M. (éds), Texte et discours en confrontation dans l'espace européen, Berne : Peter Lang.

Malrieu, D. et Rastier, F. (2001). Genres et variations morphosyntaxiques. Traitement automatique des langues, vol. 42, n², p. 548-577, http://www.revue-texto.net/Inedits/Malrieu Rastier/Malrieu-Rastier Genres.html

Marchal, H. (2009). L'ambassadeur révoqué : poésie scientifique et popularisation des savoirs au XIX ${ }^{\mathrm{e}}$ siècle. Romantisme, p. $144,25-37$.

Marcoccia, M. (2016). Analyser la communication numérique écrite. Paris : Armand Colin.

Moirand, S. (2012). Entre altération et reformulation, quelle place faire au dialogisme de Bakhtine dans les travaux de Jean Peytard ?. Synergies Monde 10 - Un hommage à Jean Peytard, Gerflint, p. 205-229.

Moirand, S. et Reboul-Touré, S. (2015). Nommer les événements à l'épreuve des mots et de la construction du discours. in Longhi J. (éd.) Langue française, 188 - Stabilité et instabilité dans la production du sens : la nomination en discours, p. 105-120.

Moirand, S., Reboul-Touré, S. et Pordeus, M. (2016). A divulgação científica no cruzamento de novas esferas de atividade linguageira, dans Bakhtiniana. Revista de Estudos do Discurso, vol. 11, no ${ }^{\circ}$ 2, São Paolo, http://revistas.pucsp.br/index.php/bakhtiniana/article/view/23847/19244

Mongi, M., Chauvin-Vileno, A. et Equoy-Hutin, S. éds. (2014). Jean Peytard, syntagmes et entailles. Actes du colloque de Besançon 7-9 juin 2012. Limoges : Lambert-Lucas, 2014.

Mortureux, M.-F. éd. (1982). Langue française - La vulgarisation 53. Paris : Larousse.

Mortureux, M.-F. (1983). La vulgarisation scientifique au XVIII siècle à travers l'œuvre de Fontenelle. Paris : Didier-Erudition.

Mounier, P. (2009). Le blogging scientifique. https://fr.slideshare.net/revuesorg/le-blogging-scientifique

von Münchow, P. (2004). Les journaux télévisés en France et en Allemagne. Plaisir de voir ou devoir de s'informer. Paris : Presses Sorbonne Nouvelle.

von Münchow, P. (2011). Lorsque l'enfant paraît... Le discours des guides parentaux en France et en Allemagne. Toulouse : PUM.

von Münchow, P. (2013). Cultures, discours, langues : aspects récurrents, idées émergentes. Contextes, représentations et modèles mentaux. in Claudel, C et alii, éds, Cultures, discours, langues : nouveaux abordages. Limoges : Lambert-Lucas, p. 186-207.

Paveau, M.-A. (2017). L'analyse du discours numérique. Paris : Hermann.

Petit Robert de la langue française. (2020).

Peytard, J. et alii. (1984). Langue française 64 - Français technique et scientifique : reformulation, enseignement. Paris : Larousse.

Peytard, J. (1993). D’une sémiotique de l'altération. Semen, 8, http://journals.openedition.org/semen/4182 
Reboul-Touré, S. (2004). Écrire la vulgarisation scientifique aujourd'hui. Colloque Sciences, Médias et Société, Lyon, ENS-LSH, $\underline{\text { http://science.societe.free.fr/documents/pdf/Sciences medias societe 2004/Reboul Toure.pdf }}$

Reboul-Touré, S. (2014). De la reformulation dans les discours de la médiation scientifique. in Madini M., Chauvin-Vileno A., EquoyHutin S. (éds), Jean Peytard : syntagmes et entailles, actes du colloque de Besançon 7-9 juin 2012, Limoges, Lambert-Lucas, p. $343-$ 353.

Reboul-Touré, S. (2015). Les blogs scientifiques francophones : aux marges de l'analyse du discours ?. in López Muñoz J. M. (éd.), Aux marges du discours. Personnes, temps, lieux, objets, actes du Xe congrès international de linguistique française, Cadix, 27-29 novembre 2013, Limoges, , Lambert-Lucas, p. 277-286.

Reboul-Touré, S. (à paraître a). Quand la reformulation s'adapte aux genres de la vulgarisation scientifique. in Ablali D., Gonçalves M. et Silva F., éds, Reformuler, une question de genre?

Reboul-Touré, S. (à paraître b). Des catégories discursives pour la comparaison ? L'exemple des blogs de vulgarisation scientifique. in Grillo V. de C. S., Glushkova M. et Reboul-Touré S., éds, Analyse du discours et comparaison : enjeux théoriques et méthodologiques.

Sepausy de, V. (2017). Démocratiser la vulgarisation scientifique en français sur internet, AUF, https://www.actualitte.com/article/patrimoine-education/democratiser-la-vulgarisation-scientifique-en-francais-sur-internet/85935

Corpus de portails de blogs autour de la vulgarisation scientifique

Agence Science presse, Québec, https://www.sciencepresse.qc.ca/blogues

Café des sciences, https://www.cafe-sciences.org/sciences-de-la-vie/

SciLogs.fr est une communauté de blogs scientifiques francophones proposée par Pour la science, http://www.scilogs.fr/

<adresses internet vérifiées le 24 03 2020>

\footnotetext{
${ }^{1}$ CEDISCOR, Centre de recherche sur les discours ordinaires et spécialisés à l'université Sorbonne nouvelle, créé en 1989 par S. Moirand. Le groupe se trouve ensuite au sein du Syled, http://syled.univ-paris3.fr/cediscor.html et se fond aujourd'hui dans l'équipe d'accueil EA 7345, Clesthia, Langage, systèmes, discours. Il est rebaptisé Cercle de recherche sur les discours, voir Les Carnets du Cediscor, https://journals.openedition.org/cediscor/.

${ }^{2}$ On note des choix différents au niveau de la nomination : on parle plus volontiers de « blog de science » au Québec et de «blog de vulgarisation scientifique » en France. Nous proposons de parler de degrés de vulgarisation scientifique (plus ou moins abordable selon les publics) ; en effet, dès que le chercheur communique vers l'extérieur, il entre dans une démarche linguistique de reformulation et s'inscrit dans « une » vulgarisation scientifique.

${ }^{3}$ Les blogs Pour la science combinent la rigueur scientifique et la convivialité des blogs. Ce site offre aux scientifiques et ceux qui s'intéressent à la science la possibilité d'interagir dans des discussions interdisciplinaires sur la science sous toutes ses formes : la recherche, les applications pratiques, l'éthique, la, politique, etc. Les blogs Pour la science sont publics : les blogs sont accessibles gratuitement à l'ensemble des internautes. (accueil : http://www.scilogs.fr/a propos/) ${ }^{4}$ En 2005, l'Agence Science-Presse a créé Science ! on blogue, le premier réseau de blogues rédigés par des scientifiques en langue française. Ce travail a été complété par un livre en octobre 2007 sur le phénomène alors naissant des blogues de science. Elle a été au cœur de deux anthologies de meilleurs blogues francophones de science, en 2013 et 2014 (https://www.sciencepresse.qc.ca/).

${ }^{5}$ L'Association francophone pour le savoir (ACFAS) anciennement l'Association canadienne-française pour l'avancement des sciences.

${ }^{6}$ Voir Les Carnets du Cediscor 1 - Un lieu d'inscription de la didacticité. Les catastrophes naturelles dans la presse quotidienne, https://journals.openedition.org/cediscor/950

${ }^{7}$ Voir Les Carnets du Cediscor 6 - Rencontres discursives entre sciences et politiques dans les médias, https://journals.openedition.org/cediscor/86

${ }^{8}$ Sur la notion de sphère empruntée à Volochinov/Bakhtine, voir Grillo 2007.
} 